\title{
Autopolypectomy of a Vocal Cord Polyp
}

\author{
Saud Ahmed, ${ }^{1}$ Altaf Hussain, ${ }^{1}$ Basharat Nadeem, ${ }^{1}$ Faroq Ali $^{1}$
}

\begin{abstract}
Introduction:
Vocal cord polyps commonly occur in those with a history of vocal abuse. Patients with large lesions generally undergo microlaryngeal surgery under general anaesthesia. This unique case report highlights a strange scenario where the patient coughed out a fleshy mass during his morning walk and which was later confirmed as a vocal cord polyp.

Case Report

A 62 year old male with a history of hoarseness of voice for 3 months presented to the ENT OPD holding a chunk of tissue which was apparently coughed out by him during his morning walk. After the incident, his symptoms had immediately improved. A videolaryngoscopy showed a congested spot on the right vocal cord being the probable site of origin of the lesion. On Histopathological examination, the tissue was reported as a vocal cord polyp. The patient was managed conservatively but the lesion recurred at the same site after a month for which a microlaryngeal excision was performed.

Discussion

Vocal cord polyps are fairly common in ENT practice and usually present to the clinic with hoarseness of voice. Polyps that are small are usually managed conservatively by voice therapy alone whereas large polyps require surgical excision. This unique case report highlights a strange clinical scenario where the patient coughed out a large vocal cord polyp (Auto-polypectomy) during a bout of acute cough. This event saved him a surgery at the first instance, but eventually had a recurrence and had to undergo an excision under $G A$.
\end{abstract}

ABSTRACT

Keywords:

Polyps; Vocal Cords

$\mathrm{V}$ ocal fold polyps are characterized by benign soft tissue swelling on medial side of vocal folds and commonly present with hoarseness of voice. They commonly arise at the junction of anterior and middle $1 / 3$ rd of the vocal fold and are commonly found in middle aged females and also seen in children due to excessive screaming or shouting. ${ }^{1}$ The most common cause is attributed to mucosal injury arising as a result of phonotrauma involving either of mucosal surfaces. These lesions inhibit the vibratory mucosal waves travelling on the vocal fold and hence patients usually present with change in the quality of voice. Depending on the size, patients can also have noisy breathing and intermittent pain. On histopathological examination, a combination of signs of recent bleeding and depositions of fibrin and iron pigment in macrophages is seen almost exclusively

1 - Department of ENT, Primecare Hospital,

Bangalore

\section{Corresponding author:}

Dr Saud Ahmed

email: saud.ahmed@primecare.in in polyps when compared with other benign lesions. ${ }^{2}$

\section{Case Report}

We report a case of a 62 year old adult male who presented with a chunk of tissue which was apparently coughed out by him during his morning walk and following which the hoarseness immediately improved. He gave a 3 month history of hoarseness of voice for which no prior medical advice was sought. Apart from being a hypertensive on regular medication there was no other significant medical history neither he had history of substance abuse. A Videolaryngoscopy showed a congested spot (raw mucosal area) with no active bleed on the superior surface of anterior $1 / 3 \mathrm{rd}$ of the right vocal cord, being the probable site of the coughed out lesion. (Fig. 1) Both the vocal cords were mobile. General physical examination along with basic laboratory investigations were within normal limits.

The coughed out tissue was sent for histopathological examination. It showed features of hyalinization, fibrinous deposits and congested vessels in the stroma 
of the lesion surrounded with multilayered squamous epithelium and the diagnosis was consistent with vocal cord polyp. (Fig. 2)

As the patient had a raw mucosal surface on the vocal cord with no active bleed, he was managed conservatively with antibiotics, antireflux medications, steam inhalation and voice rest. However, he came back after a month with hoarseness of voice. Videolaryngoscopy was repeated and showed a polypoidal and highly congested lesion on the superior aspect of the right vocal cord (anterior $1 / 3 \mathrm{rd}$ ). The lesion was crossing the midline and occupying the anterior commissure. (Fig. 3) Both vocal cords were mobile. Under antibiotic cover, he was posted for microlaryngeal surgical excision (cold steel) under general anaesthesia. The excised lesion was reported as Lobular capillary hemangioma. (Fig. 4) Postoperatively, he was advised antireflux medication, steam inhalation and absolute voice rest for 6 weeks. On follow up after 3 months he is found to be asymptomatic with no recurrence of the lesion.

\section{Discussion}

Vocal cord polyps are fairly common in ENT practice and usually present in individuals with abusive voice behaviors like excessive talking, habitual throat

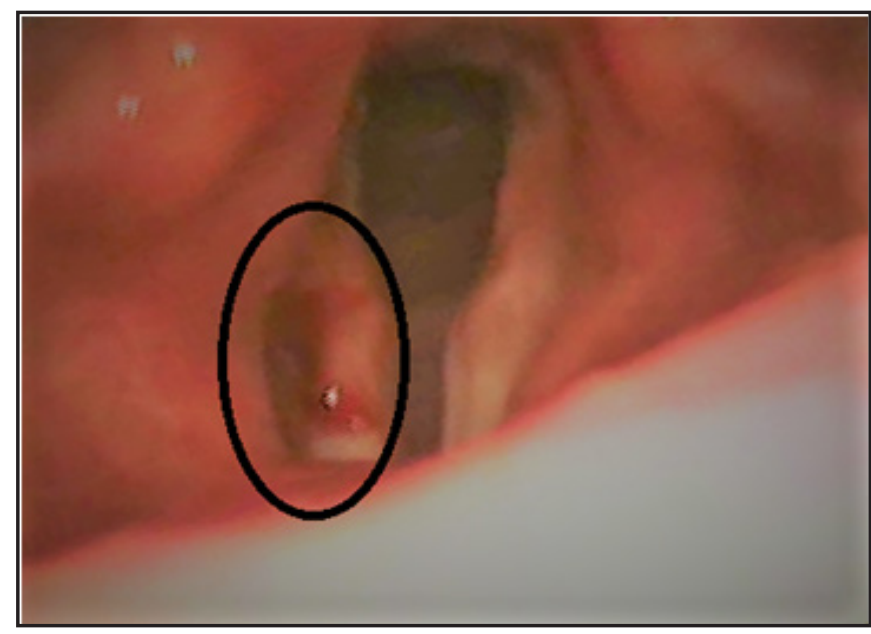

Fig. 1. Showing area of congestion in anterior $1 / 3$ rd of right vocal cord marked in oval (probable site of lesion) clearing, prolonged and excessive loudness, use of inappropriate pitch and chronic cough. Polyps start after a bout of voice trauma and present to the clinic with hoarseness of voice. ${ }^{3}$ Polyps develop at the site of maximum muscular and aerodynamic forces exerted during phonation and are considered a sequelae of phonotrauma. ${ }^{4}$ At microscopic level, there is a tear in the lamina propria and epithelium caused by shearing forces during exertion leading to capillary rupture with focal hematoma followed by inflammatory cell infiltration and new matrix formation. Polyp is formed when remodeling in these tissues happen during healing phase. They obstruct flow of mucosal waves causing hoarseness of voice. ${ }^{5}$

Polyps can present unilaterally or bilaterally and can be broad-based or pedunculated. The colour of the lesions vary and can be red, white or translucent. They can also be categorized as hemorrhagic and non-hemorrhagic types. Hemorrhagic are usually found in patient who are on anticoagulant therapy. Non-hemorrhagic polyps are found after voice abuse. ${ }^{6}$

Few investigators have hypothesized hard glottal attacks (HGA) on surfaces of vocal cords to be associated with benign lesions of vocal cords like vocal cord polyps in professional speakers, singers, and nonprofessional speakers. $^{7}$

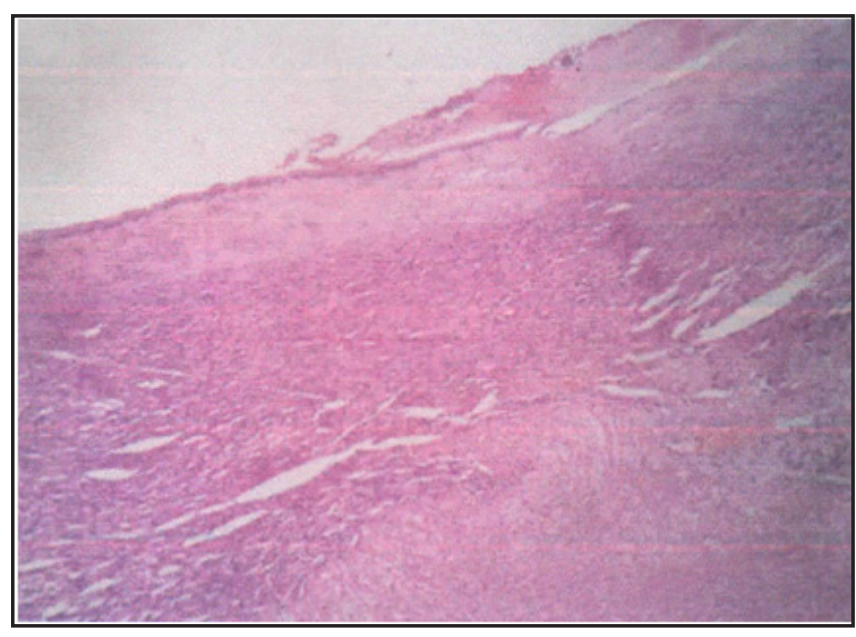

Fig. 2. HPE showing features of hyalinization, fibrinous deposits and congested vessels in the stroma of the lesion surrounded with multilayered squamous epithelium, consistent with vocal cord polyp. (H\&E, 40X) 


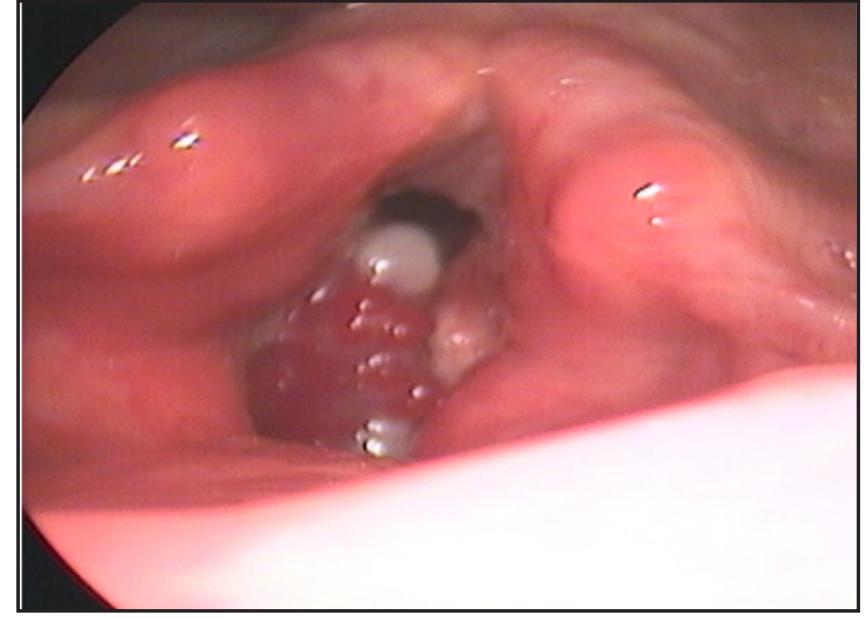

Fig. 3. Polypoidal, congested mass in anterior $1 / 3 \mathrm{~d}$ of vocal cord covering anterior commissure and crossing midline. Vocal cord movements were normal.

Rarely, patients with large polyps present with inspiratory stridor and dyspnea.

Polyps that are small are usually managed conservatively by voice therapy alone. ${ }^{8}$ Large polyps require surgical intervention. ${ }^{9}$ Voice rest, management of acid reflux and if possible, to discontinue anticoagulant for hemorrhagic polyps are usually necessary. Pulse diode laser is used to coagulate small hemorrhagic polyps. $^{10}$

Our patient miraculously had an auto-polypectomy during a bout of acute cough. This was confirmed on videolaryngoscopy findings and HPE report of the coughed out lesion. However, the lesion recurred after a month for which he had to undergo a microlaryngeal excision under GA.

\section{References}

1. Cipriani N, Martin D, Corey J, Portugal L, Caballero N, Lester $\mathrm{R}$ et al. The Clinicopathologic Spectrum of Benign Mass Lesions of the Vocal Fold due to Vocal Abuse. International Journal of Surgical Pathology 2011; 19:583-7

2. Dikkers F, Nikkels P. Benign Lesions of the Vocal Folds: Histopathology and Phonotrauma. Annals of Otology, Rhinology \& Laryngology. 1995; 104:698-703

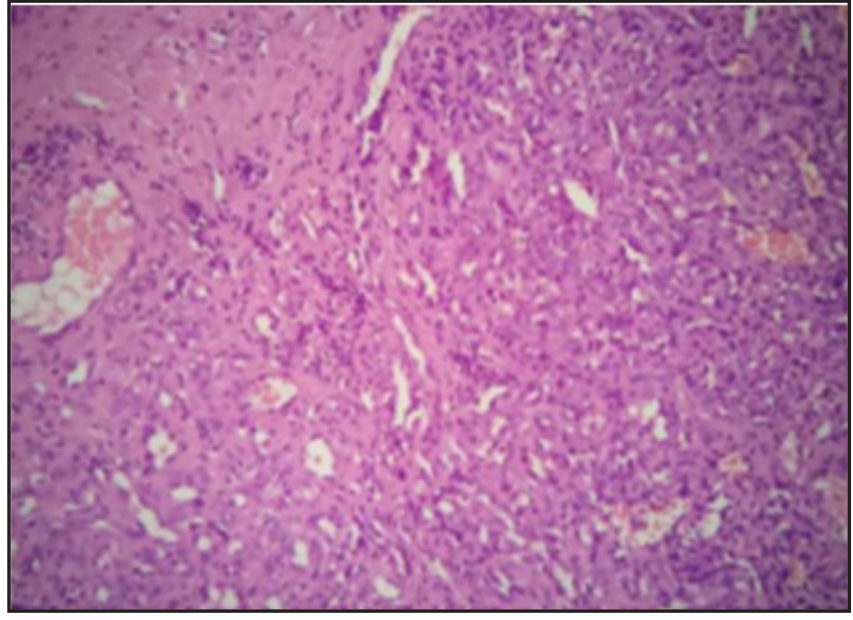

Fig. 4. HPE showing surface ulceration with fibrinous clots, proliferating capillaries and infiltrating cells suggestive of Lobular Capillary Hemangioma. Negative for malignancy. ( H\&E, 100X)

3. Bastian RW. Benign Vocal Fold Mucosal Disorders. In: Flint PW, Haughey BH, Lund VJ, et al. Eds, Cummings Otolaryngology Head and Neck Surgery, 5th Edn. Philadelphia, PA: Mosby; 2010

4. Kleinsasser O. Pathogenesis of vocal cord polyps. Ann Otol Rhinol Laryngol. 1982; 91(4 Pt 1):378-81

5. Czerwonka L, Jiang JJ, Tao C. Vocal nodules and edema may be due to vibration-induced rises in capillary pressure. Laryngoscope 2008; 118(4):748-52

6. Karkos P, McCormick M. The etiology of vocal fold nodules in adults. Current Opinion in Otolaryngology \& Head and Neck Surgery 2009; 17(6):420-3

7. Andrade D, Heuer R, Hockstein N, Castro E, Spiegel J, Sataloff $\mathrm{R}$. The frequency of hard glottal attacks in patients with muscle tension dysphonia, unilateral benign masses and bilateral benign masses. Journal of Voice 2000; 14(2):240-6

8. Schindler A, Bottero A, Capaccio P, Ginocchio D, Adorni F, Ottaviani F. Vocal Improvement After Voice Therapy in Unilateral Vocal Fold Paralysis. Journal of Voice 2008; 22(1):113-8

9. Nunes R, Behlau M, Nunes M, Paulino J. Clinical diagnosis and histological analysis of vocal nodules and polyps. Brazilian Journal of Otorhinolaryngology 2013;79(4):434-40

10. Byeon H, Han J, Choi B, Hwang H, Kim J, Choi H. Treatment of Hemorrhagic Vocal Polyps by Pulsed Dye Laser-Assisted Laryngomicrosurgery. BioMed Research International 2015; 2015:1-6. 